Ophthalmologe 2015 · 112:1023

DOI 10.1007/s00347-015-0163-y

Online publiziert: 6. November 2015

(c) Springer-Verlag Berlin Heidelberg 2015

\section{S. Abraham • N. Feucht · C.P. Lohmann - M. Maier}

Augenklinik, Klinikum rechts der Isar, München, Deutschland

\title{
Unübliche Befundkonstellation bei okulärem Ischämiesyndrom
}

\section{Erwiderung}

Zum Leserbrief von Schmidt D (2015)

Ungewöhnlicher Krankheitsverlauf bei langstreckiger A.-carotis-interna-Stenose mit okulärer Ischämie durch Verschluss der A. ophthalmica, doi: 10.1007/s00347-0150161-0

Originalbeitrag: Abraham S, Feucht N, Lohmann CP, M. Maier(2015) Okuläres Ischämiesyndrom. Ophthalmologe 112:679681. doi:10.1007/s00347-014-3178-x

Wir danken dem Autor des Leserbriefes für die interessanten Ausführungen und Nachfragen zu unserem Artikel „Okuläres Ischämiesyndrom“. Tatsächlich war die „Stolperfalle“ dieser Kasuistik die ungewöhnliche Formation der A.-carotisinterna-Stenose links, die langstreckig, jedoch nicht höhergradig (d. h. Stenosegrad weniger als $60 \%$ [1]) war und erst im Bereich der A. ophthalmica drastisch zunahm, ohne dass dies im herkömmlichen Doppler zur Darstellung kam. Die interdisziplinäre Entscheidung zur Durchführung der zielführenden Magnetresonanzangiographie erfolgte jedoch zügig, aufgrund der wegweisenden kardiovaskulären Vorgeschichte und des streng einseitigen ophthalmologischen Befundes mit schleichendem, jedoch drastischem Visusabfall über ein halbes Jahr.

Hiermit lässt sich gleichzeitig beantworten, weshalb die wichtige Differenzialdiagnose einer Riesenzellarteriitis nicht unsere primäre Verdachtsdiagnose war. Zum Ausschluss erfolgte eine Bestimmung der Blutsenkungsgeschwindigkeit sowie des Fibrinogens, welche sich als normwertig erwiesen, ebenso ließ sich keine typische Symptomatik oder ein auffälliger Tastbefund der Schläfenarterie erheben. Wir bitten zu entschuldigen, dass diese wichtigen Untersuchungen in der Publikation keine Erwähnung fanden.

Die ebenfalls wichtige Frage nach einer Seitendifferenz des Augeninnendrucks wird dahingehend beantwortet, dass „es lediglich zu zwischenzeitlich grenzwertig erhöhten Augendrücken am betroffenen Auge" kam. Dies impliziert eine gering ausgeprägte Seitendifferenz, jedoch in Richtung Hypertension des betroffenen Auges bei vorliegender Rubeosis iridis und Hyphäma und nicht in Richtung Hypotension, wie sie bei okulärer Ischämie gelegentlich gefunden wird [2].

Zusammenfassend wird erneut deutlich, welche Bedeutung einer umfassenden, gezielten Befunderhebung und Evaluation von Differenzialdiagnosen in solch einem Fall zukommt. Wir bedanken uns sehr für diesen konstruktiven Leserbrief.

\section{Korrespondenzadresse}

\section{Dr. S. Abraham}

Augenklinik, Klinikum rechts der Isar Ismaningerstraße 22, 81675 München Sophia.Abraham@mri.tum.de

\section{Einhaltung ethischer Richtlinien}

Interessenkonflikt. S. Abraham, N. Feucht, C.P. Lohmann und M. Maier geben, dass kein Interessenkonflikt besteht.

\section{Literatur}

1. Arning $C$, Widder $B$, von Reutern GM, Stiegler H, Görtler M (2010) Ultraschallkriterien zur Graduierung von Stenosen der A. carotis interna - Revision der DEGUM-Kriterien und Transfer in NASCET-Stenosierungsgrade. Ultraschall Med 31(3):251-257

2. Ruprecht KW, Naumann GOH (1979) Ischämische Ophthalmopathie - ein Leitsymptom stenosierender Karotisprozesse. 77. Zusammenkunft DOG Heidelberg 1979; 891-894 